\title{
On new arithmetic function relative to a fixed positive integer. Part 1
}

\author{
Brahim Mittou $^{1}$ and Abdallah Derbal ${ }^{2}$ \\ ${ }^{1}$ Department of Mathematics , University Kasdi Merbah, Ouargla \\ EDPNL \& HM Laboratory, ENS of Kouba, Algiers, Algeria \\ e-mails: mathmittou@gmail.com, mittou.brahimeuniv-ouargla.dz \\ ${ }^{2}$ ENS of Kouba, Algiers \\ EDPNL \& HM Laboratory, ENS of Kouba, Algiers, Algeria \\ e-mail: abderbal@yahoo.fr
}

Received: 23 June 2020

Revised: 14 November 2020

Accepted: 18 January 2021

Abstract: The main purpose of this note is to define a new arithmetic function relative to a fixed positive integer and to study some of its properties.

Keywords: Arithmetic function, Greatest common divisor.

2010 Mathematics Subject Classification: 11A25.

\section{Introduction}

Throughout this paper, we let $(a, b)$ denote the greatest common divisor of any two integers $a$ and $b$. Let

$$
n=\prod_{i=1}^{r} p_{i}^{e_{i}}
$$

be the prime factorization of the positive integer $n>1$, where $r, e_{1}, e_{2}, \ldots, e_{r}$ are positive integers and $p_{1}, p_{2}, \ldots, p_{r}$ are different primes.

In recent years, many researchers have published many papers that have been the subject of arithmetic functions (see e.g., [1-6]). In [1], Atanassov defined the following function:

$$
\underline{\operatorname{mult}}(n)=\prod_{i=1}^{r} p_{i}, \quad \underline{\operatorname{mult}}(1)=1 .
$$

The aim of this note is to define a new arithmetic function relative to a fixed positive integer $\alpha$, that can be considered a generalization of Atanassov's function and discuss some of its properties. 


\section{Main results}

Let $\alpha$ be a positive integer. Then we define $f_{\alpha}$ to be the arithmetic function such that:

$$
f_{\alpha}(n)=\prod_{i=1}^{r} p_{i}^{\left(e_{i}, \alpha\right)}, \quad f_{\alpha}(1)=1
$$

In particular, if $\alpha=1$, then $\left(e_{i}, \alpha\right)=1$ for all $(1 \leq i \leq r)$. Thus

$$
f_{1}(n)=\underline{\operatorname{mult}}(n), \quad \text { for all } n .
$$

For examples, see Table 1.

Let $m$ be a positive integer such that $m=\prod_{j=1}^{s} q_{j}^{f_{j}}$, where $s, f_{1}, f_{2}, \ldots, f_{s}$ are positive integers and $q_{1}, q_{2}, \ldots, q_{s}$ are different primes. If $(m, n)=1$ i.e., $\left(q_{j} \neq p_{i}\right.$ for all $1 \leq i \leq r$ and $1 \leq j \leq s)$, then for all $\alpha$ :

$$
f_{\alpha}(m n)=\prod_{j=1}^{s} q_{j}^{\left(f_{j}, \alpha\right)} \prod_{i=1}^{r} p_{i}^{\left(e_{i}, \alpha\right)}=f_{\alpha}(m) f_{\alpha}(n) .
$$

On the other hand, if $p_{1}, p_{2}$, and $p_{3}$ are different primes, then for all $\alpha$ :

$$
f_{\alpha}\left(p_{1} \cdot p_{2}^{2} \cdot p_{3}\right)=p_{1} \cdot p_{2}^{(2, \alpha)} \cdot p_{3}, \text { while that } f_{\alpha}\left(p_{1} \cdot p_{2}\right) f_{\alpha}\left(p_{2} \cdot p_{3}\right)=p_{1} \cdot p_{2}^{2} \cdot p_{3}
$$

Consequently, one can show that the function $f_{\alpha}$ is multiplicative but not completely multiplicative.

\begin{tabular}{|ccc||ccc||ccc||ccc|}
\hline \hline$n$ & $f_{2}(n)$ & $f_{3}(n)$ & $n$ & $f_{2}(n)$ & $f_{3}(n)$ & $n$ & $f_{2}(n)$ & $f_{3}(n)$ & $n$ & $f_{2}(n)$ & $f_{3}(n)$ \\
\hline \hline 1 & 1 & 1 & 11 & 11 & 11 & 21 & 21 & 21 & 31 & 31 & 31 \\
\hline 2 & 2 & 2 & 12 & 12 & 6 & 22 & 22 & 22 & 32 & 2 & 2 \\
\hline 3 & 3 & 3 & 13 & 13 & 13 & 23 & 23 & 23 & 33 & 33 & 33 \\
\hline 4 & 4 & 2 & 14 & 14 & 14 & 24 & 6 & 24 & 34 & 34 & 34 \\
\hline 5 & 5 & 5 & 15 & 15 & 15 & 25 & 25 & 5 & 35 & 35 & 35 \\
\hline 6 & 6 & 6 & 16 & 4 & 2 & 26 & 26 & 26 & 36 & 36 & 6 \\
\hline 7 & 7 & 7 & 17 & 17 & 17 & 27 & 3 & 27 & 37 & 37 & 37 \\
\hline 8 & 2 & 8 & 18 & 18 & 6 & 28 & 28 & 14 & 38 & 38 & 38 \\
\hline 9 & 9 & 3 & 19 & 19 & 19 & 29 & 29 & 29 & 39 & 39 & 39 \\
\hline 10 & 10 & 10 & 20 & 20 & 10 & 30 & 30 & 30 & 40 & 10 & 40 \\
\hline \hline
\end{tabular}

Table 1. The first 40 values of $f_{2}$ and $f_{3}$.

It can be easily seen that $1<f_{\alpha}(n) \leq n$ and $f_{\alpha}(n) \mid n$ for all $n>1$, since $\left(e_{i}, \alpha\right) \leq e_{i}$ for all $\alpha$. So, as a consequence $f_{\alpha}(p)=p$ for all primes $p$. The following theorem distinguishes those numbers that satisfy the equality: $f_{\alpha}(n)=n$ (for all $\alpha$ ).

Theorem 2.1. For any integer $\alpha \geq 1$, the square-free positive integers are the only integers satisfying $f_{\alpha}(n)=n$. 
Proof. Clearly, if $n$ is a square-free number, i.e., $e_{1}=e_{2}=\cdots=e_{r}=1$, then $f_{\alpha}(n)=n$. Now let $n$ be such that $f_{\alpha}(n)=n$ for all $\alpha$. Thus

$$
\left(e_{i}, \alpha\right)=e_{i} \text { for all } \alpha
$$

which is true only if $e_{i}=1$ for all $1 \leq i \leq r$, i.e., only if $n$ is a square-free number.

Corollary 2.1.1. Let $\alpha$ be a positive integer. Then for every $n$ :

$$
f_{\alpha}(\underline{\operatorname{mult}}(n))=\underline{\operatorname{mult}}(n)=\underline{\operatorname{mult}}\left(f_{\alpha}(n)\right) .
$$

Proof. It is well known that mult $(n)$ is a square-free number for every $n$, so by Theorem 2.1:

$$
f_{\alpha}(\underline{\operatorname{mult}}(n))=\underline{\operatorname{mult}}(n) .
$$

On the other hand, we have

$$
\underline{\operatorname{mult}}\left(f_{\alpha}(n)\right)=\underline{\operatorname{mult}}\left(\prod_{i=1}^{r} p_{i}^{\left(e_{i}, \alpha\right)}\right)=\prod_{i=1}^{r} p_{i}=\underline{\operatorname{mult}}(n) .
$$

Theorem 2.2. Let $\alpha$ and $\beta$ be positive integers. Then for every $n$ :

$$
f_{\alpha}\left(f_{\beta}(n)\right)=f_{(\alpha, \beta)}(n)
$$

In particular, if $(\alpha, \beta)=1$, then

$$
f_{\alpha}\left(f_{\beta}(n)\right)=\underline{\operatorname{mult}}(n) .
$$

Proof. For $n=1$, the statement is true. If $n>1$, then we have

$$
\begin{aligned}
f_{\alpha}\left(f_{\beta}(n)\right) & =f_{\alpha}\left(f_{\beta}\left(\prod_{i=1}^{r} p_{i}^{e_{i}}\right)\right) \\
& =f_{\alpha}\left(\prod_{i=1}^{r} p_{i}^{\left(e_{i}, \beta\right)}\right) \\
& =\prod_{i=1}^{r} p_{i}^{\left(\left(e_{i}, \beta\right), \alpha\right)}=\prod_{i=1}^{r} p_{i}^{\left(e_{i},(\beta, \alpha)\right)} \\
& =f_{(\alpha, \beta)}(n) .
\end{aligned}
$$

Let us suppose that $(\alpha, \beta)=1$. Then

$$
f_{\alpha}\left(f_{\beta}(n)\right)=\prod_{i=1}^{r} p_{i}^{\left(e_{i}, 1\right)}=\prod_{i=1}^{r} p_{i}=\underline{\operatorname{mult}}(n) .
$$

Theorem 2.3. Let $e=\operatorname{lcm}\left(e_{1}, e_{2}, \ldots, e_{r}\right)$. Then $f_{\alpha}(n)$ is a periodic function with period e as a function of $\alpha$, in other words:

$$
f_{\alpha+e}(n)=f_{\alpha}(n), \quad \text { for all } \alpha
$$


Proof. First of all, there exist $r$ positive integers $\left(k_{1}, k_{2}, \ldots, k_{r}\right)$ such that $e=k_{i} e_{i}(1 \leq i \leq r)$, since $e=\operatorname{lcm}\left(e_{1}, e_{2}, \ldots, e_{r}\right)$. This means

$$
\left(e_{i}, \alpha+e\right)=\left(e_{i}, \alpha+k_{i} e_{i}\right)=\left(e_{i}, \alpha\right) \quad(1 \leq i \leq r)
$$

from which, we can get

$$
f_{\alpha+e}(n)=\prod_{i=1}^{r} p_{i}^{\left(e_{i}, \alpha+e\right)}=\prod_{i=1}^{r} p_{i}^{\left(e_{i}, \alpha\right)}=f_{\alpha}(n) .
$$

Theorem 2.4. Let $\alpha$ and $\beta$ be positive integers such that $\beta=\alpha \beta^{\prime}$. If $\left(e_{i}, \beta^{\prime}\right)=1$ for all $1 \leq i \leq r$, then

$$
f_{\alpha}(n)=f_{\beta}(n)
$$

Proof. We have

$$
\left(e_{i}, \beta\right)=\left(e_{i}, \alpha \beta^{\prime}\right)=\left(e_{i}, \alpha\right)
$$

since $\left(e_{i}, \beta^{\prime}\right)=1(1 \leq i \leq r)$. Thus

$$
f_{\beta}(n)=\prod_{i=1}^{r} p_{i}^{\left(e_{i}, \beta\right)}=\prod_{i=1}^{r} p_{i}^{\left(e_{i}, \alpha \beta^{\prime}\right)}=\prod_{i=1}^{r} p_{i}^{\left(e_{i}, \alpha\right)}=f_{\alpha}(n) .
$$

Many mathematicians have been studied the perfect numbers and their generalizations with the help of various arithmetic functions (see e.g., [7, 8, 11]). In $[9,10]$, some arithmetic functions are used in characterizing generalized Mersenne primes. These primes are then used in the study of class numbers of certain number fields (see [10]). Euler showed that all even perfect numbers (EPN) are of the form $2^{p-1} m$, where $m=2^{p}-1$ is a Mersenne prime. Also, Euler stated that an odd perfect number (OPN), if it exists, must have the form $p^{e} m^{2}$, where $p$ is a prime with $(p, m)=1$ and $p \equiv e \equiv 1(\bmod 4)$.

The next theorem gives the values of $f_{2}$ for perfect numbers.

Theorem 2.5. Let $N>6$ be a perfect number. Then

$$
f_{2}(N)= \begin{cases}4 m & \text { if } N=2^{p-1} m \text { is an } E P N \\ p \cdot \underline{\operatorname{mult}}(m)^{2} & \text { if } N=p^{e} m^{2} \text { is an } O P N\end{cases}
$$

Proof. Let $N=2^{p-1} m$ be an even perfect number. Then $m=2^{p}-1$ is a Mersenne prime. But, in order for $m$ to be a prime, $p$ must itself be a prime. Thus $(p-1,2)=2$, since $N>6$, from which we have

$$
f_{2}\left(2^{p-1} m\right)=f_{2}\left(2^{p-1}\right) f_{2}(m)=2^{(p-1,2)} m=4 m .
$$

Let $N=p^{e} m^{2}$ be an odd perfect number. Then $(p, m)=1$ and $p \equiv e \equiv 1(\bmod 4)$. So $(e, 2)=1$. On the other hand, if $m=\prod_{i=1}^{r} p_{i}^{e_{i}}$, then $m^{2}=\prod_{i=1}^{r} p_{i}^{2 e_{i}}$ and $\left(2 e_{i}, 2\right)=2$ for all $(1 \leq i \leq r)$. This means

$$
f_{2}\left(p^{e} m^{2}\right)=f_{2}\left(p^{e}\right) f_{2}\left(m^{2}\right)=p^{(e, 2)} \prod_{i=1}^{r} p_{i}^{\left(2 e_{i}, 2\right)}=p\left(\prod_{i=1}^{r} p_{i}\right)^{2}=p \cdot \underline{\operatorname{mult}}(m)^{2} .
$$




\section{Conclusion}

In conclusion, we will mention, that in the second part we will study a new arithmetic function relative to a fixed positive integer $\alpha$, which will define by substituting the $\left(e_{i}, \alpha\right)$ for the $\left[e_{i}, \alpha\right]$ in (1), where $\left[e_{i}, \alpha\right]$ is the least common multiple of $\alpha$ and $e_{i}$.

\section{Acknowledgements}

The authors would like to thank the anonymous referees for their careful reading and valuable suggestions which certainly improved the readability of this paper.

\section{References}

[1] Atanassov, K. (1987). New integer functions, related to $\varphi$ and $\sigma$ functions. Bulletin of Number Theory and Related Topics, XI(1), 3-26.

[2] Atanassov, K. (2002). Restrictive factor: Definition, properties and problems. Notes on Number Theory and Discrete Mathematics, 8(4), 117-119.

[3] Atanassov, K. (2016). An arithmetic function decreasing the natural numbers. Notes on Number Theory and Discrete Mathematics, 22(4), 16-19.

[4] Atanassov, K. (2016). On function "Restrictive factor". Notes on Number Theory and Discrete Mathematics, 22(2), 17-22.

[5] Atanassov, K. \& Sándor, J. (2019). Extension factor: Definition, properties and problems. Part 1. Notes on Number Theory and Discrete Mathematics, 25(3), 36-43.

[6] Atanassov, K. \& Sándor, J. (2020). Extension factor: Definition, properties and problems. Part 2. Notes on Number Theory and Discrete Mathematics, 26(1), 31-39.

[7] Defant, C. (2018). Connected components of complex divisor functions. Journal of Number Theory, 190, 56-71.

[8] Hoque, A., \& Kalita, H. (2014). Generalized Perfect Numbers Connected with Arithmetic Functions. Mathematical Sciences Letters, 3(3), 249-253.

[9] Hoque, A., \& Saikia, H. K. (2014). On generalized Mersenne prime. SeMA Journal, 66(1), $1-7$.

[10] Hoque, A., \& Saikia, H. K. (2015). On generalized Mersenne primes and class-numbers of equivalent quadratic fields and cyclotomic fields. SeMA Journal, 67(1), 71-75.

[11] McCarthy, P. J. (1959). On an arithmetic function. Monatshefte für Mathematik, 63, $228-230$. 